Approche des versants oraux et écrits d'un genre d'exposition expert (article-conférence) dans le domaine francophone : l'écrit et l'oral soutenu(s) ont-ils les mêmes centres ?

Marie-Christine POUDER

Université René Descartes

Université Paris Ouest Nanterre La Défense

Laboratoire CNRS MoDyCo

\title{
APPROCHE DES VERSANTS ORAUX ET ÉCRITS D'UN GENRE D'EXPOSITION EXPERT (ARTICLE-CONFÉRENCE) DANS LE DOMAINE FRANCOPHONE : L'ÉCRIT ET L'ORAL SOUTENU(S) ONT-ILS LES MÊMES CENTRES ?
}

«En relisant deux lettres de Troubetskoy, que je me propose de publier en volume, j'ai été frappé par une de ses déclarations que j'avais oubliée et qui est très typique 'La syntaxe me terrifie'. Vous retrouvez la même chose, de façon très marquée, dans la linguistique française. Voyez dans le cours de Saussure, comme toutes les questions qui ne regardent plus le mot, mais déjà l'organisation de la phrase semblent troublantes. La linguistique française souffre d'une même contradiction. C'est qu'on se trouve là devant un nouveau problème, celui du discours où la liberté créative devient particulièrement grande. » ${ }^{1}$

C'est ainsi que s'exprimait en français R. Jakobson à Cambridge (Massachussets) en 1970, dans un entretien avec Jean-Pierre Faye, Jean Paris et Jacques Roubaud, entretien dans lequel il évoquait longuement ses relations avec différents cercles, en particulier ceux de Moscou, de Copenhague et de Prague.

Peu de temps auparavant, à Paris, lors d'un séminaire de J. Lacan ce même R. Jakobson signalait: ${ }^{2}$

« Si on me demande quel est le problème le plus actuel de la linguistique, le problème interdisciplinaire envers la psychologie, la psychanalyse, l'ethnologie, c'est le problème du contexte. Le contexte a deux aspects : le contexte verbalisé qui est donné dans le discours, et le contexte non-verbalisé, la situation, le contexte non-verbalisé, mais toujours verbalisable. [...] Le problème de la verbalisation joue un rôle essentiel, principal dans ces deux domaines... Si nous analysons mieux nos énonciations, nous nous apercevons que la question des citations joue le rôle primaire essentiel. L'oratio direct, l'oratio obliquae, ce sont des problèmes plus larges que la place qui leur est indiquée par la grammaire classique. C'est un des problèmes qui n'est pas encore élucidé jusqu' au bout. C'est une question que le psychologue et le linguiste doivent travailler ensemble ».

Et il poursuivait: «Mes expériences à New-York, mes rencontres avec les psychanalystes, un anthropologue comme Lévi-Strauss, moi et quelques autres linguistes, quand nous discutions nos problèmes, j'ai vu qu'il était important de

\footnotetext{
${ }^{1}$ Plus loin R. Jakobson signale L. Tesnière comme syntacticien original et Volochinov pour son introduction de la dialectique dans l'analyse du discours, in : Hypothèses, Change, Seghers / Laffont, 1972.

${ }^{2}$ La séance du séminaire de J. Lacan (La logique du Fantasme, 1966-67) du $1^{\text {er }}$ février 1967 est une véritable séance autour de la personnalité de R. Jakobson, soumis au feu roulant des questions de J. Lacan et d'autres participants Lacaniens (J. Aubry, L. Irigaray, Dr. Oury, M. Médèze, Dr. Stoianoff). La dernière question de J. Lacan portait sur les rapports de maître à disciple que Jakobson élude en partie en personnalisant le moins possible et en faisant l'éloge de l'interdisciplinarité.
} 
devenir pour un instant le disciple de ces autres disciplines pour voir la langue de dehors comme on voit la terre de dehors en montant dans un spoutnik ».

En France, la psychanalyse lacanienne, la revue Change sensibilisaient un public assez ouvert aux problématiques, déjà anciennes, du Cercle de Prague. La valeur programmatique des Thèses de 1929 a favorisé leur extension hors de leur contexte local relevant de l'étude spécifique des langues slaves. En tant que coconstructeur de ces thèses, R. Jakobson fit alors figure avec quelques autres linguistes plus ou moins intégrés dans les institutions universitaires (Benveniste, Barthes, Bakhtine, Martinet) de démystificateur des perspectives strictement néogrammairiennes confinées dans le commentaire logiciste en boucle d'exemples quasi immémoriaux.

Passer de la phrase au discours ne s'est pas fait simplement, même si la demande sociale du moment aidait fortement les jeunes linguistes à se plonger dans le bain de leur(s) langue(s) pour y mettre le discours de leurs maitres à l'épreuve.

A cette époque, un professeur de linguistique, spécialiste de la phonologie du français à l'Université de Paris V (D. François) a eu la surprise de constater, lors d'une interview à France Culture que les exemples oraux fournis pour l'entretien avaient été «nettoyés » par les ingénieurs du son pour les rendre conformes aux critères d'audibilité de la chaîne. Les éléments rejetés, pauses, hésitations, répétitions, épenthèses constituaient en fait tous les points qui seront ensuite mis en observation par les chercheurs. La linguistique semblait pourtant bien destinée à étudier l'oral, mais il s'agissait d'un oral fictif, reconstruit à partir de notes et de questionnaires, transcrit, à une période où l'aide logicielle n'était pas encore disponible au royaume du binarisme structuraliste triomphant.

A la fin des années 70, J. Goody, un spécialiste de sociétés africaines «sans écriture » a fait partie de ceux qui ont commencé à poser les vraies questions sur l'oralité, il signalait : ${ }^{3}$

"L'idée que le langage parlé et le langage écrit puissent être de nature distincte a rencontré des résistances chez bien des linguistes. [...] Contre cette tendance générale, nous trouvons le courant théorique représenté par certains chercheurs liés au Cercle Linguistique de Prague et nommé par eux ' fonctionnaliste ' : J. Vachek en particulier, dit que ' coexistent, à l'intérieur d'un seul et même langage, deux normes, celle de l'écrit et celle du parlé' ; [...] L'écriture est utilisée à des fins spécialisées et souvent culturellement plus valorisée (à cela s'ajoute l'imprimerie, qui ' dépersonnalise ' l'énoncé écrit); c'est pourquoi, selon Vachek, dans l'opposition entre les deux normes, c'est la norme écrite qui est le terme ' marqué ' ». En ce sens, elle joue un rôle de régulateur dans les changements linguistiques.

C'est ainsi qu'entre 1950 et 1977 se sont positionnés des courants de recherche qui, faisant jouer l'interdisciplinarité entre littérature et sciences humaines ont influencé notablement, en partie sous l'égide des théoriciens praguois, la recherche sur les aspects réalistes et pragmatiques des différents discours constituant une langue.

\footnotetext{
${ }^{3}$ GoODY Jacques (1979).
} 
Approche des versants oraux et écrits d'un genre d'exposition expert (article-conférence) dans le domaine francophone : l'écrit et l'oral soutenu(s) ont-ils les mêmes centres ?

En reprenant les idées avancées dans ces quelques références introductives, il est possible de saisir une certaine régularité dans l'exercice de la liberté créative du discours, tant à l'oral qu'à l'écrit. L'étude systématique des contextes non verbalisés ainsi que l'analyse fine de la verbalisation et des fonctions remplies par les énoncés permet de cerner des genres et des sous-genres définis par des chronotopes et des mouvements énonciatifs qui entretiennent entre eux des rapports complexes de filiation par le biais des citations et des répétitions. Il est possible d'interroger la dualité des normes à partir de quelques situations témoins.

\section{Une situation contemporaine}

A Paris, durant l'année 2000, l'année du passage au nouveau millénaire, la communauté savante francophone a suscité un événement au long cours, la tenue journalière d'une conférence de spécialité, enregistrée et mise en ligne sur internet, en général dans un amphithéâtre parisien. Ces conférences ont ensuite donné lieu à une version écrite dans une collection de poche. ${ }^{4}$

Le genre dont il relève est proche du cours universitaire par sa durée et par le lieu où il se tient; un animateur présente brièvement un conférencier qui s'exprime sur un sujet durant une cinquantaine de minutes et qui répond ensuite aux questions du public. La mise en relation des enregistrements et de la version écrite permet de mettre en évidence un effet de continuum entre des formes différenciées d'oral et d'écrit, ce qui permet d'approcher en synchronie, par rapport à un thème donné et pour un même locuteur, des effets de variations. Un continuum relie essentiellement cinq formes d'expression: oral dialogal / oral monologal / oral scripturalisé / écrit oralisé / écrit publié/. Ces formes correspondent à des cadrages situationnels différents.

L'oral dialogal correspond à l'oral utilisé par l'animateur, par le conférencier et par le public lors de la présentation et lors de l'échange questions-réponses en fin de conférence. Dans ces situations se retrouvent les formes habituellement considérées comme les plus « relâchées » de la langue pour un individu.

L'oral monologal correspond à l'oral utilisé par les conférenciers improvisateurs qui, en l'occurrence, sont également des utilisateurs de moyens audio-visuels.

L'oral scripturalisé correspond en partie à l'oral d'improvisateurs qui font visualiser des transparents avec des éléments textuels, mais surtout à l'oral de lecteurs qui s'éloignent sans cesse de leur texte initial sur un empan faible, pour faire des micro- ou macro-commentaires.

L'écrit oralisé correspond à une lecture assez fidèle d'un texte préalablement écrit avec quelques grands commentaires et excursus oraux libres.

L'écrit publié correspond au texte publié dans le livre de poche, produit éditorial rendant finalement compte de la conférence.

Il serait possible de gloser longtemps sur les différentes façons de comparer l'oral et l'écrit, sur l'opportunité ou la nécessité de le faire, ainsi que sur l'absurdité de tenter de mettre en relation des phénomènes qui sont de toute manière en relation de continuité et / ou de complémentarité. Il est néanmoins

\footnotetext{
${ }^{4}$ Version écrite de l'UTLS 2000, vingt volumes aux éditions O. Jacob, 2001-2002.
} 
Approche des versants oraux et écrits d'un genre d'exposition expert (article-conférence) dans le domaine francophone : l'écrit et l'oral soutenu(s) ont-ils les mêmes centres ?

important de rendre compte des différents types de réécriture (orales ou écrites) en cause, à la lumière en particulier de travaux sur les genèses textuelles, la reformulation ou le discours rapporté. L'étude de deux versions d'un « même » événement constitue en fait une sorte de fiction d'une comparaison oral / écrit en discours expert.

La focalisation sur certains points de détail permettra d'introduire un biais pour opposer fonctionnement oral et/ou écrit dans cette situation complexe.

\section{Problème d'unités}

L'automatisation des procédures d'analyse lexicale sur ordinateur a privilégié comme critère de délimitation de l'écrit le segment situé entre une majuscule et un point ; ce critère déjà problématique pour l'écrit est totalement irréaliste pour l'oral, particulièrement en ce qui concerne le monologue improvisé.

Ce type d'analyse produit bien sûr des résultats..., à l'écrit ou sur un corpus d'oral transcrit, retravaillé et préparé. Ainsi après un rapide nettoyage des textes scannés des articles, un logiciel d'analyse des co-occurrents (Alceste) privilégiant une approche lexicale classifie les douze conférences qui constituent ce corpus de travail. La conférence du médecin s'oppose à toutes les autres, les articles de trois psychologues sont regroupés, ainsi que ceux de deux sociologues ; un sociologue qui s'exprime sur l'illettrisme utilise un lexique proche de celui d'un pédagogue expert en sciences de l'éducation. Les deux psychanalystes ne sont pas réunis par leur lexique, l'un s'exprimant plutôt de manière théorique et l'autre évoquant des histoires de cas; une anthropologue de la famille et un sociologue travaillant sur les âges de la vie ont chacun des formulations assez spécifiques.

L'exemple suivant met en relation les versions orale et écrite d'un fragment initial de l'intervention du linguiste B. L.

Oral: (00:02:00) Comme point d'départ euh, on peut partir d'une constatation extrêm'ment simple qui concerne la communication. Bien, si on s'intéresse à..., la communication, un circuit d'la communication, on peut dire qu'les choses sont, euh, d'une grande simplicité : un locuteur A émet un certain signal sonore, un certain message, ce message est réceptionné par un locuteur $B$, ce locuteur $B$ reçoit l'information. C'est donc une..., un système extrêm'ment simple en apparence, simplement quand on s'intéresse à ce circuit de façon un peu plus précise, on voit immédiat'ment qu'un grand nombre de problèmes sont posés et qu'les choses sont beaucoup moins simples que ce qu'on pourrait penser à..., priori ou à première vue quand on s'intéresse à la communication. (00:02:55)

Ecrit: Le circuit de communication qui s'établit entre un locuteur et son interlocuteur est, en apparence, simple : un message est émis par A, il est reçu par $B$ et une information s'est échangée entre A et B. En fait, dès que l'on analyse plus précisément ce circuit, les événements apparaissent beaucoup plus complexes.

L'oral accentue rhétoriquement les effets d'annonce et de clôture, de reprise à distance et généralise les effets de bouclage, les éléments métadiscursifs et les effets d'énonciation. Il y a souvent une inversion dans l'ordre d'apparition des éléments thématiques du discours. On observe aussi des éléments de répétition, de chaînage, des effets d'insistance ou de modalisation. Le potentiel paraphrastique de la situation joue sur certaines transformations (actif / passif, positif / négatif). Il 
Approche des versants oraux et écrits d'un genre d'exposition expert (article-conférence) dans le domaine francophone : l'écrit et l'oral soutenu(s) ont-ils les mêmes centres ?

est net que la différence d'unité à l'oral, chez les improvisateurs absolus (paragraphe vs énoncé), dépend aussi du dispositif audiovisuel qui a des incidences sur les plans hiérarchiques, syntaxiques et énonciatifs.

Les exemples suivants mettent également en regard des versions orales et écrites de fragments de conférences.

Le premier exemple illustre la gestion de la lecture d'un lecteur de son texte, sociologue auteur d'un livre sur «Les âges de la vie» (X. G.); le locuteur ne projette aucun document mais balise sa lecture d'éléments qui rendent sa présentation très phatique par rapport au public.

Oral : Donc, on peut dire, d'une certaine façon, sans crainte de s'tromper que la France, par rapport à d'autres pays (00:19:34), elle a les étudiants les plus âgés et les r'traités les plus jeunes. Donc, vous voyez tous les problèmes déjà que ça pose pour notre sujet. Alors là aussi quelques chiffres; le taux d'activité des hommes en France, de 55 à 64 ans, est en France le... le plus bas de tous les pays,... il est de $44 \%$, alors que au Royaume-Uni et aux Etats-unis, il est de...euh 20, de 20 points de plus, $64 \%$ et $66 \%$ et que en Suède et au Japon, il est de 40 ans....de 40 points supérieur $(00: 20: 10)$, que par ailleurs, si on,...on compare la France aux Etats-Unis, on s'aperçoit, et le chiffre est quand même extrêmement surprenant, je pense, pour vous, que le taux d'chômage est,... enfin le,... disons plutôt le taux d'activité est plus faible aux Etats-Unis qu'en France pour les gens entre 30 et 50 ans, c'qui montre bien que tout l'problème de l'emploi en France, enfin..., la plus grande partie, elle porte sur les jeunes et les plus âgés (00 : 20. : 39). Si j'prends un autre chiffre, la sortie de la vie professionnelle est d'plus en plus précoce, de 62 ans en 1969 à 58 ans maint'nant, et l'entrée dans cette même vie professionnelle, elle est de plus en plus tardive; en 1969, c'était à 18 ans, tandis que maint'nant en 97, c'est à 22 ans.

Ecrit : La France a les étudiants les plus âgés et les retraités les plus jeunes. Le taux d'activité des hommes de 55 à 64 ans est en France le plus bas de tous les pays comparables (OCDE) : $44 \%$ alors qu'il est de 20 points supérieur au Royaume-Uni et aux Etats-Unis (soit $64 \%$ et $66 \%$ ) et de 40 points supérieurs en Suède et au Japon (80,5\% et $85 \%$ ). La sortie de la vie professionnelle est de plus en plus précoce: de 62 ans en 1969 à 58 ans maintenant. Et l'entrée dans cette même vie professionnelle est de plus en plus tardive : de 18 ans (1969) à 22 ans (1997).

Sur le plan fonctionnel nous observons ici une implication marquée du locuteur dans son énonciation (je / vous; vous / je; on), des éléments de focalisation, de clivage et de reprise pronominales, des incises, des hésitations et des «phrases » plus longues à l'oral qu'à l'écrit. L'orateur parsème sa lecture de segments relatifs à la programmation métadiscursive de ce qu'il a déjà mentionné ou de ce qui lui reste à dire.

Le second exemple est illustratif d'une opposition totale ou partielle entre version orale et version écrite de la conférence : une partie est développée à l'oral et pas à l'écrit (l'auteur «profite» du contact avec le public pour faire des révélations), ou le contraire (l'auteur prévient le public que le sujet sera abordé à l'écrit), ou simplement les exemples sont différents à l'oral et à l'écrit (exemples 
cliniques, expériences). Ainsi, la linguiste Cl. B. B. évoque dans son article un exemple qui n'est pas développé dans sa conférence

Ecrit : La linguistique descriptive se présente comme utile. Elle servira par exemple à décrire et à stabiliser le tchèque littéraire ou, dans les années 1930, à montrer l'égalité entre toutes les langues en réfutant les doctrines racistes développées à l'époque en Scandinavie et en Allemagne.

Le cadrage oral ou écrit entraîne chez les auteurs des prises de décision relatives à l'ordonnancement entre le tout et les parties de leur intervention.

Le troisième exemple met en scène la plus jeune des conférencières, une psycholinguiste qui présente les écarts maximaux en morphosyntaxe entre version écrite et version orale de la conférence. Il est examiné avec l'idée sous-jacente que ce qui est dit, qui parait contraire à une certaine conception de l'économie dans la langue, n'est pas dit fortuitement mais semble nécessaire à ce moment-là pour le locuteur mis dans cette situation en fonction de ses objectifs de communication et de son expertise sur le sujet.

Oral (improvisation, commentaire de transparent): Donc, y'a pas simplement cet argument purement formel, grammatical, qui indique que / une partie de la grammaire est innée, mais y'a aussi tout un tas d'autres faits qu'on peut observer dans la nature; donc le premier, c'est que le langage est spécifique à l'homme. Donc, d'une part, tous les êtres humains parlent...

Ecrit : Outre cet argument formel, d'autres faits suggèrent que la capacité à apprendre une langue humaine est innée. Le langage est spécifique à l'homme. D'une part, tous les êtres humains parlent...

Toute une suite de connecteurs, de quantificateurs disparaissent à l'écrit au profit de phrases courtes à sujets nominaux. Des spécifications, une restriction et une amplification, prises de position en faveur des sciences de la nature et de l'observation d'un grand nombre de faits, sont «gommées » à l'écrit.

L'exemple est limité, mais ce travail est sans cesse à l'œuvre et produit ainsi peu à peu un effet réductionniste qui transforme un texte argumenté, circonstancié, modalisé et explicatif en thèse rigide et autoritaire.

\section{Quelques figures d'usage à l'oral et à l'écrit}

\section{La négation}

Le morphème discontinu «(ne)... pas » est la négation verbale la plus utilisée, plus de 1350 fois dans le corpus avec une forte différence entre l'oral et l'écrit (en moyenne utilisé 7 fois plus à l'oral avec des variations de 2 à 26), souvent lié à une modalisation. L'oral lu conserve intégralement l'aspect discontinu, l'oral monologique encore très majoritairement, alors que l'oral conversationnel généralise l'abandon de la discontinuité. Seule la plus jeune des conférencières a un plus fort taux d'élision en commentant ses transparents $(50,8 \%)$ qu'à l'oral conversationnel.

Les variations âge, mode de présentation et peut-être nationalité (deux conférenciers sont belges d'origine) sont actives.

Ce point est à mettre en relation avec l'absence du «ne » dans l'acquisition du langage. La négation par «non» ou par «pas» (pas dodo, pas fini, pas toi, 
non....) est très précoce chez l'enfant, encore très fortement liée à des gestes de refus ou d'opposition, à des intonations forcées; la non-apparition à l'oral conversationnel du signifiant discontinu chez les adultes même lettrés entretient bien son absence chez le jeune locuteur qui ne rencontrera le phénomène stabilisé qu'à l'écrit (lecture répétée d'histoires, apprentissage de l'écriture à partir de phrases de style écrit).

La négation de ce type est également assez utilisée à l'écrit puisque $17 \%$ des énoncés entre majuscule et point contiennent au moins une négation. Sur le plan signifié la négation soutient un ensemble de procédés qui dessinent un univers rhétorique particulier (contradiction, binarisation, balancements) et évitent la répétition (tournures paraphrastiques, antonymes). ${ }^{5}$

\section{L'interrogation}

L'utilisation de l'interrogation n'est pas liée à l'oral dialogique. La formulation interrogative intervient régulièrement comme balisage, titrage, à l'écrit, ou encore comme focalisation à valeur métadiscursive, annonce d'un développement, introduction d'un paradigme nouveau dans la discipline, rupture et reformulation métalinguistique à $1^{\text {'oral }}{ }^{6}$ : huit pour cent en moyenne des énoncés entre majuscule et point contiennent une interrogative. Chez quelques locuteurs et particulièrement chez la plus jeune des intervenantes, l'opposition entre fonctionnement oral et fonctionnement écrit est maximale.

Oral Conf. A. Ch. : C'est quoi la nature de ce qui doit être appris ? (1) On peut s'demander, c'est quoi exactement qui est inné? (12) C'est quoi les phonèmes de la langue? C'est les catégories sonores hein? (13) Qu'est-ce que c'est ces règles qui permettent de calculer le sens des mots hein ? (3) Ils doivent dire est-ce que Oscar a dit des choses correctes ou pas des choses correctes. (6) Comment on fait pour tester ça? (25) - Comment on fait pour leur donner la tournure syntaxique? (29) Il reste combien de temps? (27)

Ecrit Conf. A. Ch. : Comment les enfants apprennent-ils les règles syntaxiques? (5) Comment ont-ils fait pour apprendre une règle aussi complexe aussi rapidement? (6) Pourquoi cette observation est-elle si importante? (9) Qu'est-ce qui est inné exactement? (11) Quelle est la cause de l'accent étranger? (16) D'où vient cette complexité ? (10)

Dans les exemples oraux nous ne notons aucune inversion du sujet dans l'interrogative, aucune expression claire du discours indirect qui est traité comme un discours direct rapporté, une généralisation d'une forme en «c'est quoi ? ».

Ces faits sont peut-être à mettre partiellement en relation avec un usage fréquent de l'anglais chez cette locutrice ou avec l'attitude d'hypocorrection de certains intellectuels anciennement notée par les sociolinguistes, mais ils sont congruents avec les formes utilisées par de jeunes élèves non anglophones en français contemporain. Certaines formes précoces, si elles ne sont pas relevées et corrigées, perdurent dans le parler des jeunes enfants : «c'est quoi vous faites? Devine c'est quoi ?» (enfant de trois ans ou jeune dysphasique de neuf ans). Ces

\footnotetext{
${ }^{5}$ POUdER (2008b)

${ }^{6}$ POUdER (2006)
} 
formes apparaissent souvent chez l'enfant de trois ans avant des questionnements en «qu'est-ce que.... » ou en «ce que c'est... ? ».

L'absence d'inversion du sujet à l'oral est bien partagée par les improvisateurs ; les locuteurs lecteurs de leur texte et non-utilisateurs de moyens de projection, mobilisent eux, souvent avec emphase, l'inversion du sujet, au besoin même dans des tournures non-interrogatives ou l'inversion n'est pas habituelle (Là encore ne se voit aucune contrainte qui procèderait d'un fondement naturel...Anthropologue Fr. H.-A.).

\section{Passivation}

Durant les année 1970, de très nombreuses grammaires scolaires et universitaires ont inscrit sur leurs pages des arbres de transformation en tentant de démontrer la parenté ou la plus ou moins grande proximité cognitive de structures du type affirmatif/négatif, déclaratif/interrogatif, actif/passif, à partir d'analyses faites initialement sur l'anglais et ses structures à auxiliaires. Elles ont entraîné de manière adultocentriste les enfants à produire des énoncés hautement improbables en assimilant l'usage langagier à une sorte de puzzle logico-mathématique et à un jeu formel d'oppositions.

Il faut plus que jamais contester ce type exclusif de présentation de phénomènes linguistiques qui sont parfois liés à des acquisitions très précoces (négation, opposition, refus, intonation de questionnement, pseudo-dialogue) ou qui renvoient à des maîtrises plus lentes (actif, affirmatif sans aspect ou modélisation) voire très lentes à s'installer (passivation, discours indirect).

Dans ces corpus le passif (de phrase, à différencier d'autres types de passivation) est très peu utilisé dans son expression maximale avec expression d'agent ; 5\% des énoncés écrits comportent un passif, taux qui est encore plus faible à l'oral (en moyenne avec de grosses variations idiolectales, par exemple le passif peut être utilisé à l'oral chez ceux qui ne l'utilisent pas à l'écrit). Il semble que les lecteurs de leur texte utilisent le plus de passifs de ce genre.

Si certaines langues omettent presque toujours l'agent (chinois, arabe,...), il n'est guère certain non plus que le français l'exprime très souvent sinon dans des genres discursifs très spécifiques (langue juridique, informations, presse évènementielle, écrits scientifiques). Ce n'est pas ici une structure de retournement et de paraphrase habituelle.

- Ce syndrome a été décrit pour la première fois par Léo Kanner en 1943. (T.N.8-Or-éc); Cet enseignement est impulsé par un..., par le professeur. (T.N.41-Or) ; il a été trouvé récemment par une équipe de japonais que...(O.H.23Or).

- Les bases ont été décrites par la neurochimie traditionnelle (P.C. 25-éc); Des enfants... peuvent avoir été pris par des djinns. (T.N.13-Or-éc); On est conduit par des habitudes discursives. (B.Lah.4-Or).

- ...bien que ses formes ne soient pas dictées par la nature... (Fr.H-A.22-éc); Cette image va être traduite par cette matrice de pixels (P .C .5-Or); les relations sont marquées par une forte et durable dépendance (FrH-A.20-Or).

Les exemples sont présentés à dessein de manière à glisser sémantiquement de formes nettement «passivées » avec agent humain et complément(s) repérables à 
d'autres usages qui mobilisent la structure agentive lexicale plus à titre formel que sémantique. L'expression développée du passif conduit soit à un rationalisme exacerbé soit à une vision du monde poétique et métaphorique, qui est somme toute celle du langage ordinaire avec ses scories de rationalités lointaines et de schématisations récentes, dans un glissement constant du côté du moyen ou de l'instrumental. La reformulation à l'oral met en évidence des sortes de paires minimales avec changement de préposition, adverbiation ou passage au moyen.

Face à ce petit nombre de "passifs» développés, un grand nombre de déverbatifs sont utilisés, ainsi que de nombreux participes-adjectifs à valeur passive avec ou sans mention d'agent, indices de la portée souvent infraphrastique du sémantisme passif (...la découverte des rayons X par Röntgen - la première expérience sur la perception de la parole par les bébés, cette appropriation de la fécondité par les hommes...)

Ces structures sont assez caractéristiques de ce mode d'exposition de vulgarisation qui, tout en se voulant explicatif, ne peut donner la raison de toutes choses. Imaginons Gulliver débarquant dans un pays où tous les passifs seraient suivis d'agents clairement définis et actualisés, un pays où toute action recevrait une explication déterministe précise, soit scientiste, soit mythologique, ou encore poétique, ce qui, en discours, est presque la même chose mais n'a vraiment pas les mêmes incidences cognitives.

Les longs développements des astrophysiciens sur l'évolution des systèmes de galaxies ne nous empêchent pas de regarder le soleil se lever et se coucher sans remettre en cause nos expressions habituelles. Selon une logique strictement scientifique il faudrait sans cesse changer d'expressions en fonction des modifications des recherches et des systèmes explicatifs. Le niveau d'abstraction de certaines unités de la langue est en fait lié à leur utilisation par décontextualisation des procès, présentant les concepts comme de purs états interagissant les uns avec les autres, masquant des couches d'intermédiaires et d'opérateurs dans les abîmes du discours.

De même que le médecin ne peut expliquer et imager que jusqu'à un certain point le fonctionnement d'un scanner à émission de positons ou le métabolisme du cerveau dans ce contexte de vulgarisation, le sociologue ou le psychiatre utilisent des concepts externes issus de l'œuvre de Foucault ou de Deleuze, qui construisent un monde délimitant un univers de croyance plus ou moins consensuel pour des groupes restreints.

En ce sens une partie des usages du passif en discours signalés dans « La grammaire d'aujourd'hui ${ }^{7}$ se retrouvent dans ce corpus (discursif, agentif, connectif, thématique).

«Les usages du passif en discours... (permettent) de ne pas exprimer l'identité du responsable du procès..., de rétablir un ordre préférentiel sujet animé / complément inanimé ;... de faciliter les ellipses, de faciliter la liaison avec une autre phrase, de faire porter l'attention sur l'information qui paraît primordiale au locuteur...».

\footnotetext{
${ }^{7}$ ArRIVE Michel, GADET Françoise, GALMICHE Michel (1986)..
} 
Les auteurs signalent également des tournures, particulièrement fréquentes dans ces conférences, des formes pronominales à sens passif ou moyen.

- ...Le développement du cerveau s'effectue par espèces de vagues - l'enfant se laisse guider par sa perception - ce chiasme s'explique... par le sort différent réservé aux deux sexes - l'évolution qui se joue sous nos yeux...

Toutes ces formes apparaissent assez tardivement dans l'acquisition du langage et dépendent de l'inculcation d'un type de lexique/culture bien représenté dans les langues romanes.

\section{Conclusion}

Toutes ces différences ciblées entre usage écrit et usage oral pour un genre donné relèvent-elles uniquement de codages diversifiés, voire de registres variés ou tendent-elles à s'organiser autour de deux réseaux régulés d'affinités normatives comme pourraient le suggérer les résultats des travaux sur les littératures orales?

Pour reprendre l'image du continuum, les habitudes de codage de l'oral improvisé se différencient très nettement de celles de l'article publié (thématisation en boucle, ordonnancement et répétition, modalisation, fonctions du langage dominantes, morpho-syntaxe variable, types d'énoncés à actualisateurs particuliers...); mais en ce qui concerne les termes médians du continuum (oral scripturalisé, écrit oralisé), les différences semblent davantage relever des fonctions pragmatiques assumées (confidence, prise à témoin, exemplification, reformulation métalinguistique).

La mise en relation de ces faits avec les faits d'acquisition chez les enfants avant 6 ans permettrait peut-être de mieux sérier ce qui relève précisément de deux normes de mise en mots (culture de l'oral vs culture de l'écrit) ou de ce qui relèverait d'un noyau central pragmatico-sémantique recevant des expansions en périphérie l'engageant fortement dans des restructurations progressives.

\section{BIBLIOGRAPHIE}

ARrivé M., Gadet F., Galmiche M., (1986), La grammaire d'aujourd'hui, guide alphabétique de linguistique française, Flammarion.

Change $n^{\circ} 3$, (1969), Le Cercle de Prague, Seghers / Laffont.

GoODY J., (1979), La raison graphique : la domestication de la pensée sauvage, coll. Le sens Commun, Les Editions de Minuit.

Hypothèses, (1972), Trois entretiens et trois études sur la linguistique et la poétique, série Hypothèses, Change, Seghers / Laffont. "Entretien de R. Jakobson avec J-P. Faye, J. Paris, J. Roubaud».

LACAN J., (1981), La logique du fantasme, séminaire 1966-67, Editions du Piranha, Paris.

POUDER M.-CH., (2006), Dialogie constitutive, formelle ou fictive dans l'exposition de vulgarisation (conférences UTLS), Colloque International Interaction et pensée : perspectives dialogiques, Université de Lausanne. Mis en ligne sur le site hal.archives-ouvertes.fr. 
Approche des versants oraux et écrits d'un genre d'exposition expert (article-conférence) dans le domaine francophone : l'écrit et l'oral soutenu(s) ont-ils les mêmes centres ?

POUDER M.-CH., (2008a), Quel français comme langue de spécialité ? Hétérogénéité des modes d'exposition et de vulgarisation du savoir à l'oral et à l'écrit, in: Actes du XXXI ${ }^{\text {ème }}$ colloque International de Linguistique fonctionnelle (Lugo, septembre 2007), Editorial AXAC.

POUDER M.-CH., (2008b), De l'oral de la conférence à l'écrit de l'article, figures de la négation dans un genre de vulgarisation scientifique, Actes du Colloque International, La négation en discours (Sousse-Kairouan, avril 2008). Mis en ligne sur le site : [http://hal.archives-ouvertes.fr/aut/Pouder].

\section{SUMMARY}

Confrontation between spoken and written versions of French-speaking experts conferences makes possible to approach the problem of a mono or doubly centred language, according to an analysis introduced by some theorists of the Prague's Circle.

We underline the existence of a continuum in the forms of speech linked to the different domains exercised by the speakers as well as their manners to communicate (reading paper, free speaking, showing slides).

The distant forms in the continuum (monologue improvisation/ written paper) are opposed in many major aspects in putting words (topics-order, detachments, variations in morphosyntax, types of actualisation), the median forms (scripturalized speaking, oralized writing) seem to differ in pragmatic functional oppositions. 